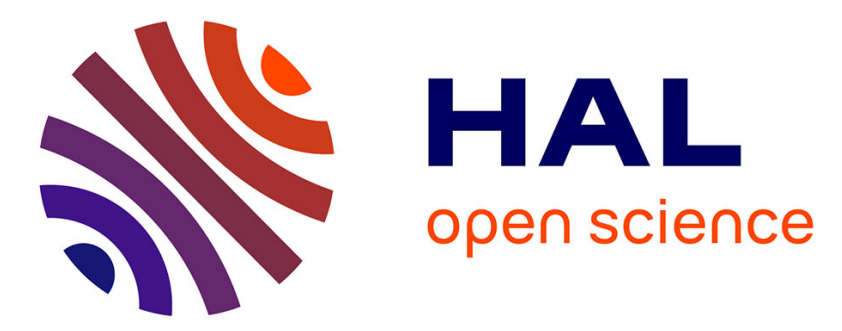

\title{
Cooperative Self-Assembly of Photochromic Diarylethenes at Liquid/Solid Interface and Highly Sensitive Photoinduced Transformation of the Ordering
}

Kenji Matsuda, Takashi Hirose, Soichi Yokoyama, Denis Frath

\section{- To cite this version:}

Kenji Matsuda, Takashi Hirose, Soichi Yokoyama, Denis Frath. Cooperative Self-Assembly of Photochromic Diarylethenes at Liquid/Solid Interface and Highly Sensitive Photoinduced Transformation of the Ordering. Photon-Working Switches, pp.409-419, 2017, 978-4-431-56542-0. 10.1007/978-4-43156544-4_20. hal-01916218

\author{
HAL Id: hal-01916218 \\ https://hal.science/hal-01916218
}

Submitted on 8 Nov 2018

HAL is a multi-disciplinary open access archive for the deposit and dissemination of scientific research documents, whether they are published or not. The documents may come from teaching and research institutions in France or abroad, or from public or private research centers.
L'archive ouverte pluridisciplinaire HAL, est destinée au dépôt et à la diffusion de documents scientifiques de niveau recherche, publiés ou non, émanant des établissements d'enseignement et de recherche français ou étrangers, des laboratoires publics ou privés. 


\title{
Chapter \$
}

\section{Cooperative Self-Assembly of Photochromic Diarylethenes at Liquid/Solid Interface and Highly Sensitive Photoinduced Transformation of the Ordering}

\author{
Kenji Matsuda, Takashi Hirose, Soichi Yokoyama, and Denis Frath \\ Department of Synthetic Chemistry and Biological Chemistry, Graduate School of \\ Engineering, Kyoto University, Katsura, Nishikyo-ku, Kyoto 615-8510, Japan \\ e-mail: kmatsuda@sbchem.kyoto-u.ac.jp
}

\begin{abstract}
In this Chapter, self-assembly of photochromic diarylethene at liquid/solid interface and photoinduced transformation of the ordering are described. By the use of scanning tunneling microscopy, the process of assembly can be studied at the molecular resolution. By the measurement of concentration dependence of surface coverage and the introduction of cooperative adsorption model, the degree of cooperativity in the self-assembly process can be evaluated and the guiding principle for highly sensitive photoresponsive system can be obtained. It is demonstrated that the precise control of the self-assembly process on $2 \mathrm{D}$ surface becomes possible by the careful design of the molecular structure.
\end{abstract}

Key words: Photochromism; Diarylethene; Scanning Tunneling Microscopy; SelfAssembly; Cooperativity

\section{\$.1 Introduction}

By the advances of technologies in fabrication and manipulation of nano-scale structure, handling of organic molecule has become possible recently. By making the devices from organic molecule, electroconductive and photophysical properties of single molecule can be utilized in the fields of molecular-scale electronics and photonics.[1-3] By the way, photochromic molecules, which show reversible photoinduced structural change, are considered as a promising candidate for molecular-scale switching device.[4] Among several photochromic molecules, diarylethene derivative show reversible photoinduced change of electronic structure 
from the viewpoint of $\pi$-conjugation. Therefore, they are considered to work as a molecular-scale switch in molecular electronics.[5]

However, in order to use photochromic molecules practically in molecular electronics, assembly and arrangement of the molecules are very important. Therefore, it is very important to develop methodologies to align molecules on a twodimensional surface. This chapter describes our recent achievements on the photocontrol of the self-assembled ordering composed of photochromic diarylethenes at liquid/solid interface investigated by scanning tunneling microscopy (STM). This technique, originally developed by Rabe et al.,[6] is attracting interests because it can visualize the process of molecular assembly at the molecular resolution.

Two-dimensional crystal engineering at liquid/solid interfaces has attracted growing attention in the last decade.[7-11] To efficiently design and predict the formation of thermodynamically stable molecular ordering formed through selfassembly processes, interplays of various interactions, such as molecule-substrate interactions, intermolecular interaction,[12-15] solvent effects,[16-19] and the balance of enthalpy and entropy terms,[20-23] must be taken into account. The stimuli-responsive interface and the control of the phase transition is also attracting interest.[24,25] In this Chapter we will focus on the use of the cooperative self-assembly to design highly sensitive stimuli-responsive assembly.

\section{\$.2 Self-Assembly and Photoinduced Transformation of Molecular Ordering of Photochromic Diarylethenes at Liquid/Highly Oriented Pyrolytic Graphite (HOPG) Interface} [26]

Diarylethene-pyrene diad $\mathbf{1}$ and diarylethene-pyrene-diarylethene triad $\mathbf{2}$ were synthesized to investigate the photoinduced two-dimensional ordering change at a solution/highly oriented pyrolytic graphite (HOPG) interface by STM. The long alkyl chain and pyrene were introduced into the diarylethene in order to stabilize the 2D structure. Pyrene interacts with HOPG due to extended planar $\pi$ conjugation. The alkyl chains are also effective at interacting with the HOPG substrate.

The different photochromic isomers showed different orderings reflecting the differences in their molecular structures. For the diarylethene-pyrene-diarylethene triad 2, a new ordering appeared upon irradiation with UV light to the ordering of open-open isomer 2(0-0) and returned to the original ordering upon subsequent irradiation with visible light at octanoic acid/HOPG interface (Fig. \$.1). The new ordering was assigned to the ordering of the closed-closed isomer 2(c-c) by comparing the image with the images of the isolated open-closed isomer 2(o-c) and the closed-closed isomer 2(c-c).

Because the adlayer is a monomolecular layer, most of the molecules exist in the solution rather than at the interface. Therefore, most of the photochromic reac- 
tions take place in the solution not at the interface. When the contents of the solution phase are thereby changed, adsorption and desorption processes exchange molecules between the interface and the solution. The UV irradiation should give both the open-closed isomer 2(o-c) and the closed-closed isomer 2(c-c), but the ordering of 2(o-c) was not observed. In order to examine the relative magnitudes of the adsorption process, we measured the STM image of the mixture with the ratio of o-o:o-c:c-c $=45: 33: 22$. The image predominantly consisted of the closedclosed isomer 2(c-c) ordering. This implies that 2(o-0) and 2(o-c) are less likely to be physisorbed on the HOPG surface than 2(c-c).The difference in the affinity to the substrate is thought to regulate the ordering behavior.

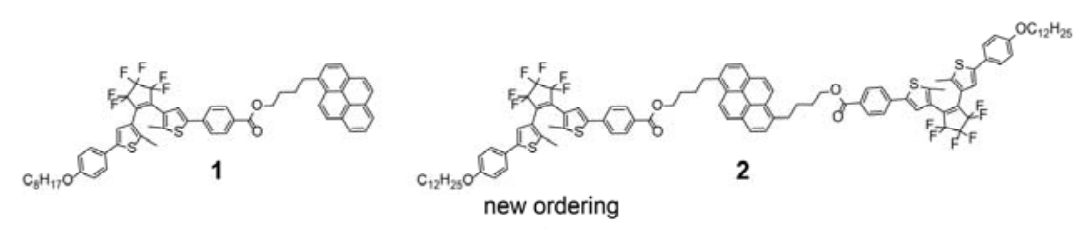

(a)

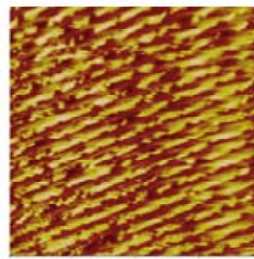

(b)

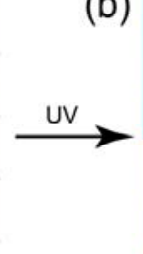

$\uparrow$

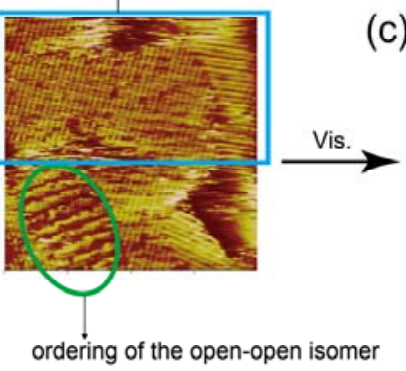

(c)

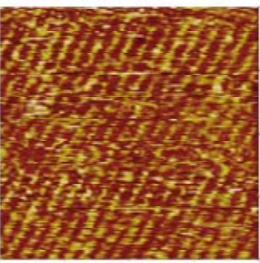

ordering of the open-open isome

Fig. \$.1. Sequence of STM images at the interface of octanoic acid-HOPG upon UV and visible irradiation of compound $\mathbf{1}(\mathbf{0}-\mathbf{0})\left(100 \times 100 \mathrm{~nm}^{2}\right.$, the same areas of the sample): (a) before UV irradiation, $\left.I_{\text {set }}=10 \mathrm{pA}, V_{\text {bias }}=-2.0 \mathrm{~V}\right)$; (b) after $U V$ irradiation for $5 \mathrm{~s}\left(I_{\text {set }}=10 \mathrm{pA}, V_{\text {bias }}=-2.5 \mathrm{~V}\right)$; (c) after successive visible irradiation $\left(I_{\mathrm{set}}=10 \mathrm{pA}, V_{\text {bias }}=-2.5 \mathrm{~V}\right)$. Adapted with permission from [26]. Copyright 2008 American Chemical Society.

\section{\$.3 Self-Assembly and Photoinduced Transformation of Molecular Ordering of Diarylethenes Carrying Amide Group} [27]

As a next step, we investigated diarylethene 3 carrying amide group that forms hydrogen bond. Diarylethene 3 having two amide groups formed characteristic stripe structures at the 1-octanoic acid/HOPG interface as expected (Fig. \$.2), while no ordering was observed with compounds having ester group instead of amide group under the same condition. The result clearly suggests that amide 
group acts as an interacting group to stabilize molecular ordering via intermolecular H-bonding network.

The width of the bright rows was $3.5 \mathrm{~nm}$ for 30 (ordering $\alpha$ ) and $1.5 \mathrm{~nm}$ for 3c (ordering $\beta$ ), suggesting different ordering patterns for the two isomers. The difference in the arrangement would be attributed to the rigid core framework of the closed-ring isomer whose delocalized $\pi$-conjugation system prefers to form faceto-face $\pi-\pi$ stacking assembly. Although the structural transformation from the ordering $\beta$ to the ordering $\alpha$ was reasonably induced by in situ visible light irradiation, in situ UV irradiation resulted in an entirely different third ordering $\gamma$ no matter whether initial ordering was $\alpha$ or $\beta$.

In order to reveal the origin of ordering $\gamma$, the annulated isomer 3a was photochemically synthesized on a milligram scale by UV irradiation to a solution of $\mathbf{3 o}$ overnight and subsequently isolated by HPLC. The ordering of 3a was confirmed to be identical to the ordering $\gamma$ that was observed after in situ UV irradiation. From these results, we concluded that the ordering $\gamma$ is composed of the annulated isomer 3a. The reason why the ordering $\beta$ was not observed upon in situ UV irradiation is the strong preferential formation of ordering $\gamma$, which may be attributed to the rigid and highly symmetric structure of the annulated isomer. The control over three states was possible in this photochromic molecule.

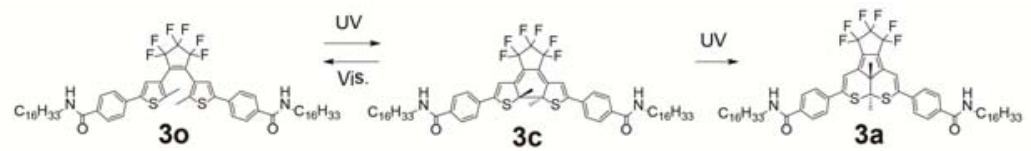

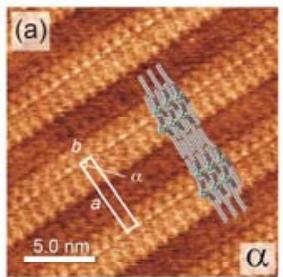

(d)

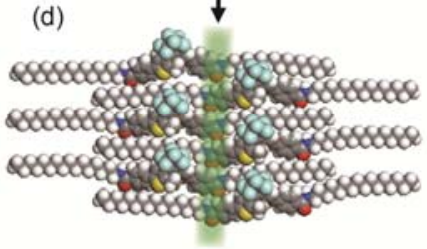

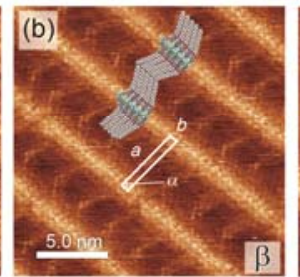

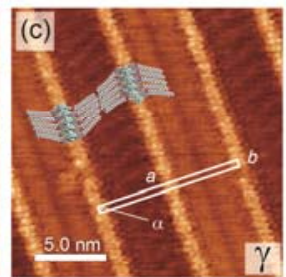

(e)

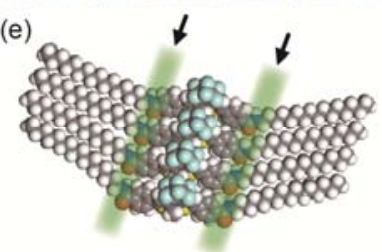

Fig. \$.2. STM images of (a) the open-ring isomer 30 (the ordering $\alpha, I_{\text {set }}=60 \mathrm{pA}, V_{\text {bias }}=-1.0 \mathrm{~V}$, $a=5.71 \mathrm{~nm}, b=0.89 \mathrm{~nm}, \alpha=88^{\circ}$ ), (b) the closed-ring isomer $3 \mathrm{c}$ (the ordering $\beta, I_{\text {set }}=20 \mathrm{pA}$, $V_{\text {bias }}=-0.9 \mathrm{~V}, a=4.82 \mathrm{~nm}, b=0.56 \mathrm{~nm}, \alpha=85^{\circ}$ ) at the 1-octanoic acid-HOPG interface. (c) The ordering after UV irradiation for $15 \mathrm{~min}$ (the ordering $\gamma, I_{\text {set }}=30 \mathrm{pA}, V_{\text {bias }}=-1.0 \mathrm{~V}, a=10.2$ $\mathrm{nm}, b=0.48 \mathrm{~nm}, \alpha=87^{\circ}$ ). (d) Enlarged molecular model of the open-ring isomer 30 and (e) that of the closed-ring isomer 3c. Hydrogen-bond network of amide groups is highlighted in green. Adapted with permission from [27]. Copyright 2013 The Chemical Society of Japan. 


\section{\$.4 Cooperative Self-Assembly of 2-Thienyl-Type Diarylethene and Photoinduced Transformation of the Ordering [28]}

In order to further develop photocontrollable assembly at liquid/solid interface, we have introduced cooperative model on the analysis of adsorption process of a 2-thienyl-type diarylethene derivative 4 (Fig. \$.3). The open-ring isomer 4o, having an amide group, formed uniform stripe-patterned molecular ordering. The ordering pattern was successfully reproduced by a molecular modeling study with the molecular mechanics/molecular dynamics (MM/MD) approach. It is revealed that a parallel conformer of the open-ring isomer is stabilized by intramolecular hydrogen bonding between the embedded two amide groups.

The concentration dependence of surface coverage showed abrupt increase at around $200 \mathrm{mM}$. Meanwhile, no ordering was observed for the closed-ring isomer 4c at various concentrations. Inspired by the cooperative self-assembly in solution, we developed a cooperative self-assembly model on a 2D surface on the basis of the Langmuir adsorption model incorporating two different equilibrium constants, the nucleation constant $K_{\mathrm{n}}$ and the elongation constant $K_{\mathrm{e}}$ (Fig. \$.4).[29] The elongation constant $K_{\mathrm{e}}$ and the degree of cooperativity $\sigma=K_{\mathrm{n}} / K_{\mathrm{e}}$ were obtained as $4600 \pm 20 \mathrm{M}^{-1}$ and $(5 \pm 2) \times 10^{-4}$, respectively. The high cooperativity was found to be the origin of the experimentally observed abrupt change of surface coverage over concentration in a supernatant solution.

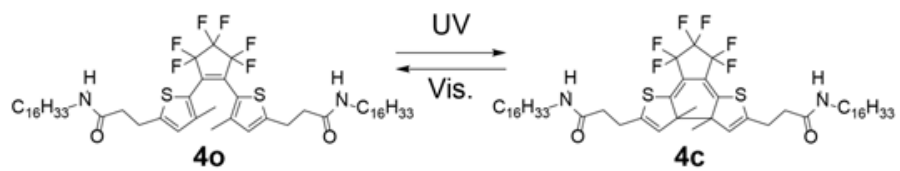

(a)

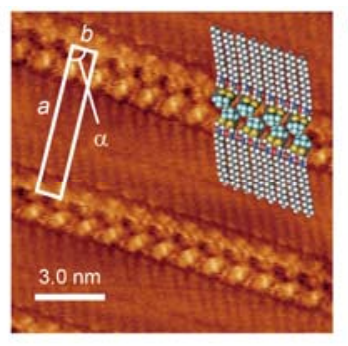

(b)

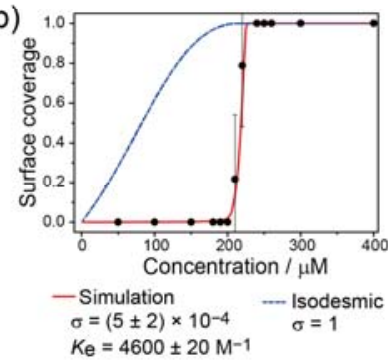

$4 \mathrm{c}$

$$
\begin{gathered}
\theta=(1-\theta) \frac{\sigma K_{\mathrm{e}}\left(c_{\mathrm{t}}-\alpha \theta\right)}{\left\{1-K_{\mathrm{e}}\left(c_{\mathrm{t}}-\alpha \theta\right)\right\}^{2}} \\
\alpha=\frac{A_{\text {sub }}}{L \cdot N_{\mathrm{A}} \cdot S}
\end{gathered}
$$

Fig. \$.3. (a) STM image of the ordering of 40 at the octanoic acid/HOPG interface $(c=250 \mathrm{mM}$, $I_{\mathrm{set}}=30 \mathrm{pA}, V_{\text {bias }}=800 \mathrm{mV}, a=6.3 \pm 0.2 \mathrm{~nm}, b=1.04 \pm 0.04 \mathrm{~nm}, \alpha=88.6 \pm 0.91^{\circ}$ ). (b) Concentration dependence of the fractional coverage of 4o. The solid line denotes the best-fit curve by the model simulation $\left(K_{\mathrm{e}}=4600 \pm 20 \mathrm{M}^{-1}, \sigma=(5 \pm 2) \times 10^{-4}\right)$ and the dashed line denotes the simulated curve with an isodesmic model $(\sigma=1)$. Adapted with permission from [28]. Copyright 2014 The Royal Society of Chemistry.

This result suggests that reversible formation/disappearance of molecular ordering is possible by means of photoisomerization between 40 and 4c. We evalu- 
ated the relationship between the surface coverage and conversion ratio of 4 . As expected, the surface coverage showed a rapid decrease at certain conversion ratio, at which the concentration of $\mathbf{4 0}$ in a supernatant solution shows the abrupt change of the surface coverage. Interestingly, the critical concentration of $\mathbf{4 0} \mathrm{ob}-$ served from the photoirradiation experiment corresponds well to the simple dilution experiment, suggesting that the presence of the closed-ring isomer $\mathbf{4 c}$ did not influence the ordering formation of $\mathbf{4 0}$. As a consequence, highly sensitive photocontrol over the cooperative $2 \mathrm{D}$ ordering formation/disappearance process on the surface is possible.

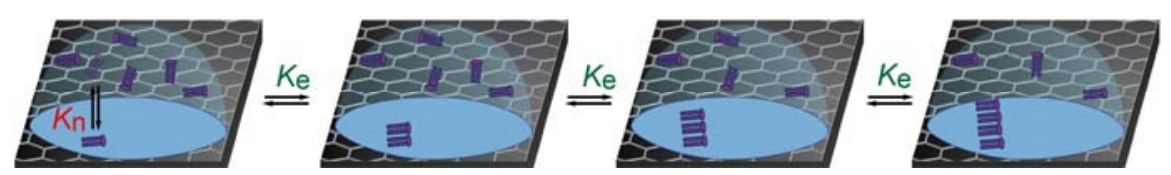

Fig. \$.4. Schematic drawing of cooperative self-assembly at a liquid/solid interface. $K_{\mathrm{n}}$ denotes nucleation equilibrium constant and $K_{\mathrm{e}}$ denotes elongation equilibrium constant.

\section{\$.5 Effects of Alkyl Chain Length and Hydrogen Bonds on the Cooperative Self-Assembly of 2-Thienyl-Type Diarylethene [30]}

In the previous section, we have described the cooperative self-assembly of diarylethene molecules at liquid/solid interface. To investigate the relationship between molecular structure and the mechanism of cooperative ordering formation, we synthesized 2-thienyl-type diarylethenes 5o-Cn (5o-C16 is equivalent to 4o) bearing various lengths of alkyl side chains linked via an amide group. The concentration dependence of the surface coverage was analyzed using a cooperative model for a 2D surface based on two characteristic parameters, nucleation equilibrium constant $\left(K_{\mathrm{n}}\right)$ and elongation equilibrium constant $\left(K_{\mathrm{e}}\right)$ (Fig. \$.5).

As the length of the alkyl chain of diarylethene increased, the $K_{\mathrm{e}}$ value increased exponentially, although the pattern of molecular ordering did not change. This behavior resulted from enthalpy gain with increasing alkyl chain length, which was associated with the concentration dependence of micelle formation. For diarylethenes 6o-Cn, in which amide groups at the root of the alkyl chain were replaced by ester groups, the cooperative parameter, $\sigma$, approached unity and the mechanism resembled an isodesmic process. This was likely to be due to the absence of hydrogen-bonding interactions for the ester derivative. In terms of the effect of core structure for the ester derivatives of 6o-C22 and 6c-C22, the $\sigma$ value of the closed-ring isomer $\mathbf{6 c - C 2 2}$ was significantly smaller than that of the openring isomer 6o-C22, but the $K_{\mathrm{e}}$ values were similar. This suggests that $K_{\mathrm{e}}$ values of diarylethenes $\mathbf{6 0 -} \mathbf{C 2 2}$ and $\mathbf{6 c - C} 22$ are regulated by dispersion interactions between the alkyl chain and HOPG substrate, irrespective of the core structure; on the other 
hand, $K_{\mathrm{n}}$ was significantly affected by the core structure. The energy gain for nucleation on substrates for the closed-ring isomer $\mathbf{6 c - C 2 2}$ was lower than that of the open-ring isomer 6o-C22, which gave rise to a large difference in cooperative self-assembly on the HOPG surface.

In conclusion of this section, it is demonstrated that the precise control of selfassembly process on 2D surface becomes possible by the careful design of the molecular structure.
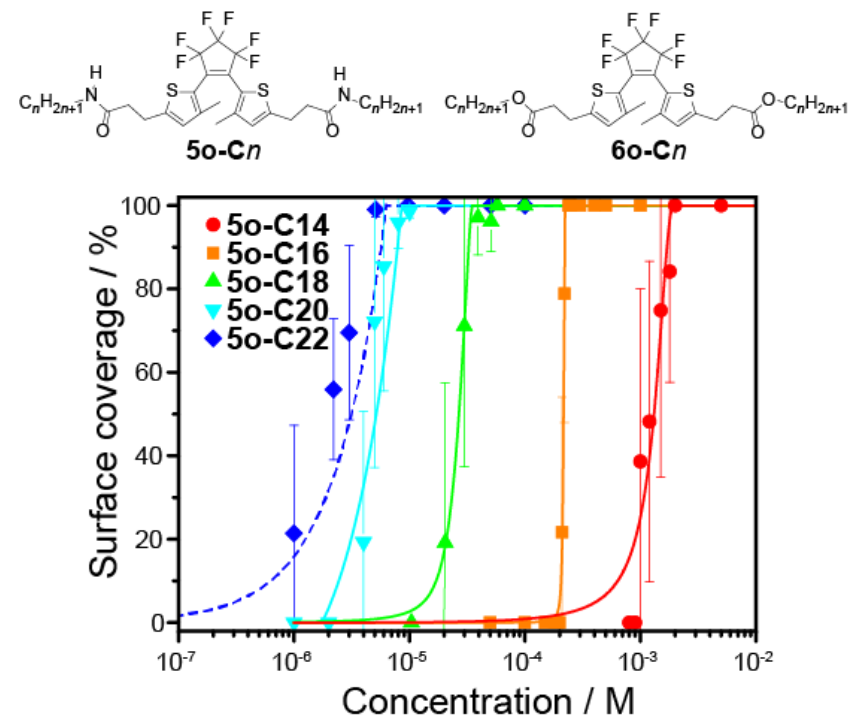

Fig. \$.5. Concentration dependence of surface coverage of the amide derivatives 5o-C14 (circle), 5o-C16 (square), 5o-C18 (upward-pointing triangle), 5o-C20 (downward-pointing triangle), and 5o-C22 (diamond) at the octanoic acid/HOPG interface. Solid lines denote the curves of best-fit by the cooperative equilibrium model. The dashed line denotes the curve based on theoretical maximum for a monolayer. Adapted with permission from [30]. Copyright 2015 Wiley-VCH Verlag GmbH \& Co. KGaA.

\section{\$.6 Cocrystallization and Mixing-Induced Cooperativity [31]}

The adsorption behavior of amide-containing diarylethene 3 was revisited by using cooperative adsorption model. The nucleation $\left(K_{\mathrm{n}}\right)$ and elongation $\left(K_{\mathrm{e}}\right)$ equilibrium constants were determined by the analysis of concentration dependence of surface coverage. It was found that the annulated isomer has a very large equilibrium constant, which explains the predominantly observed ordering of the annulated isomer (Fig. \$.6a). 
The effect of the presence of 30 on the formation of ordering $\beta$ is seen in Fig. $\$ .6 \mathrm{~b}$, where surface coverage of ordering $\beta$ is plotted against the concentration of 3c. If the formation of ordering $\beta$ is independent of the presence of the open-ring isomer 3o, the same concentration dependence is expected for any ratio of isomers (3o/3c). However, at a given concentration of 3c, the surface coverage of ordering $\beta$ is significantly increased by addition of 3o. This suggests that the ordering $\beta$ is likely a mixed crystal composed of the two isomers.

Upon mixing the closed-ring isomer 3c to ordering $\alpha$ that is mainly composed of the open-ring isomer 3o, a steep rise emerged in the concentration dependence of surface coverage (Fig. \$.6c). The prominent increase of the cooperativity was observed. It is suggested that the presence of 3c induces cooperativity into the formation process of molecular ordering composed of 3o. A quantitative analysis of the formation process of ordering using the cooperative model has provided a new view of the formation of $2 \mathrm{D}$ multicomponent molecular ordering.

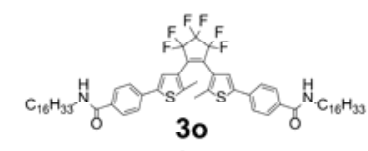

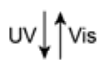
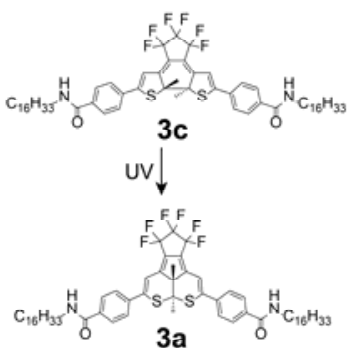

(a)

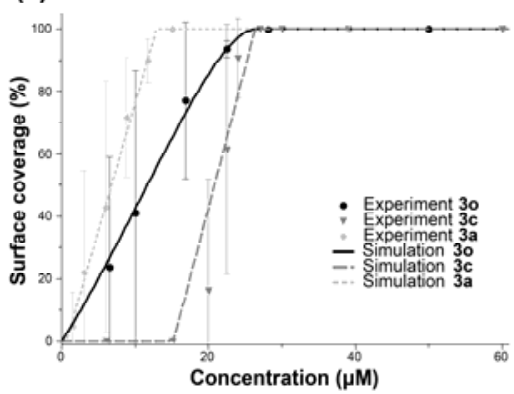

(b)

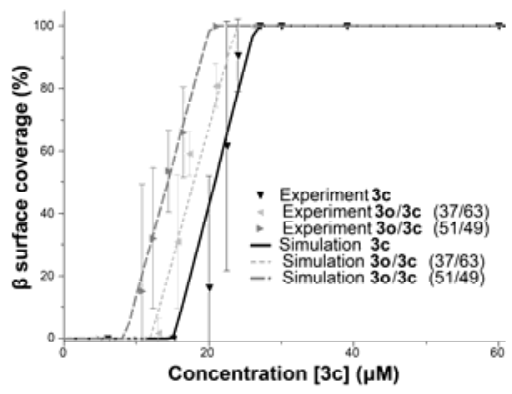

(c)

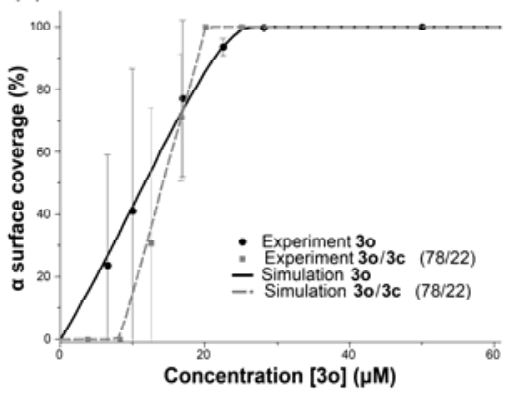

Fig. \$.6. (a) Concentration dependence of the surface coverage of 3o, 3c, and 3a at the octanoic $\mathrm{acid} / \mathrm{HOPG}$ interface. Concentration dependence of the surface coverage of (b) ordering $\beta$ for $3 \mathrm{c}$ and mixtures of 3o/3c (37:63 and 51:49) and of (c) ordering $\alpha$ for 3o and amixture of 3o/3c (78:22). Adapted with permission from [31]. Copyright 2015 Wiley-VCH Verlag GmbH \& Co. $\mathrm{KGaA}$. 


\section{Conclusion}

We have demonstrated the quantitative study of self-assembly process of photochromic diarylethene at liquid/solid interface by scanning tunneling microscopy. By the measurement of the concentration dependence of the surface coverage and the introduction of cooperative adsorption model, the degree of cooperativity of self-assembly process can be evaluated and the guiding principle for highly sensitive photoresponsive system can be obtained. These results provided a significant amount of information on the self-assembly of photochromic molecules for the application to molecular-scale electronics and photonics.

Acknowledgments: This research was supported by the Funding Program for Next Generation World-Leading Researchers (NEXT program, no. GR062) and a Grant-in-Aid for Scientific Research on Innovative Areas "Photosynergetics" (no. 26107008) from the Ministry of Education, Culture, Sports, Science and Technology (MEXT), Japan.

\section{References}

1 Barth JV, Costantini G, Kern K (2005) Engineering atomic and molecular nanostructures at surfaces. Nature 437:671-679

2 Joachim C, Ratner MA (2005) Molecular electronics: some views on transport junctions and beyond. Proc. Natl. Acad. Sci. U.S.A. 102:8801-8802

3 Moth-Poulsen K, Bjørnholm T (2009) Molecular electronics with single molecules in solidstate devices. Nat. Nanotechnol. 4:551-556

4 Feringa BL, Browne WR (Eds) (2011) Molecular switches 2nd ed. Wiley-VCH, Weinheim

5 Irie M, Fukaminato T, Matsuda K, Kobatake S (2014) Photochromism of diarylethene molecules and crystals: memories, switches, and actuators. Chem. Rev. 114:12174-12277

6 Rabe JP, Buchholz S (1991) Commensurability and mobility in two-dimensional molecular patterns on graphite. Science 253:424-427

7 Palma, CA, Cecchini M, Samorì P (2012) Predicting self-assembly: from empirism to determinism. Chem. Soc. Rev. 41:3713-3730

8 Elemans JAAW, Lei S, De Feyter S. (2009) Molecular and supramolecular networks on surfaces: from two-dimensional crystal engineering to reactivity. Angew. Chem. Int. Ed. 48:7298-7332

9 De Feyter S, De Schryver FC (2003) Two-dimensional supramolecular self-assembly probed by scanning tunneling microscopy. Chem. Soc. Rev. 32:139-150

10 Xue Y, Zimmt MB (2012) Patterned monolayer self-assembly programmed by side chain shape: four-component gratings. J. Am. Chem. Soc. 134:4513-4516

11 Nath KG, Ivasenko O, MacLeod JM, Miwa JA, Wuest JD, Nanci A, Perepichka DF, Rosei F (2007) Crystal engineering in two dimensions: an approach to molecular nanopatterning. J. Phys. Chem. C 111:16996-17007

12 Zhang X, Zeng Q, Wang C (2013) Molecular templates and nano-reactors: two-dimensional hydrogen bonded supramolecular networks on solid/liquid interfaces. RSC Adv. 3:1135111366

13 Cyr DM, Venkataraman B, Flynn GW (1996) STM Investigations of organic molecules physisorbed at the liquid-solid interface. Chem. Mater. 8:1600-1615 
14 Tahara K, Katayama K, Blunt MO, De Feyter S, Tobe Y (2014) Functionalized surfaceconfined pores: guest binding directed by lateral noncovalent interactions at the solid-liquid interface. ACS Nano 8:8683-8694

15 Xu L, Miao XR, Ying X, Deng WL (2012) Two-dimensional self-assembled molecular structures formed by the competition of van der Waals forces and dipole-dipole interactions. J. Phys. Chem. C 116:1061-1069

16 Yang Y, Wang C (2009) Solvent effects on two-dimensional molecular self-assemblies investigated by using scanning tunneling microscopy. Curr. Opin. Colloid Interface Sci. 14:135147

17 Destoop I, Ghijsens E, Katayama K, Tahara K, Mali KS, Tobe Y, De Feyter S (2012) Solvent-induced homochirality in surface-confined low-density nanoporous molecular networks. J. Am. Chem. Soc. 134:19568-19571

18 Xu L, Miao X, Zha B, Deng W (2012) Self-assembly polymorphism: solvent-responsive twodimensional morphologies of 2,7-ditridecyloxy-9-fluorenone by scanning tunneling microscopy. J. Phys. Chem. C 116:16014-16022

19 Takami, T, Mazur U, Hipps KW (2009) Solvent-induced variations in surface structure of a 2,9,16,23-tetra-tert-butyl-phthalocyanine on graphite. J. Phys. Chem. C 113:17479-17483

20 Blunt MO, Adisoejoso J, Tahara K, Katayama K, Van der Auweraer M, Tobe Y, De Feyter S (2013) Temperature-induced structural phase transitions in a two-dimensional self-assembled network. J. Am. Chem. Soc. 135:12068-12075

21 Song W, Martsinovich N, Heckl WM, Lackinger M (2013) Born-Haber cycle for monolayer self-assembly at the liquid-solid interface: assessing the enthalpic driving force. J. Am. Chem. Soc. 135:14854-14862

22 Miyake Y, Nagata T, Tanaka H, Yamazaki M, Ohta M, Kokawa R, Ogawa T (2012) Entropycontrolled 2D supramolecular structures of N,N'-bis(n-alkyl)-naphthalenediimides on a HOPG surface. ACS Nano 6:3876-3887

23 Gutzler R, Sirtl T, Dienstmaier JF, Mahata K, Heckl WM, Schmittel M, Lackinger M (2010) Reversible phase transitions in self-assembled monolayers at the liquid-solid interface: temperature-controlled opening and closing of nanopores. J. Am. Chem. Soc. 132:5084-5090

24 Ciesielski A, Palma CA, Bonini M, Samorì P (2010) Towards supramolecular engineering of functional nanomaterials: pre-programming multi-component $2 \mathrm{D}$ self-assembly at solidliquid interfaces. Adv. Mater. 22:3506-3520

25 Zhang XM, Zeng QD, Wang C (2013) Reversible phase transformation at the solid-liquid Interface: STM reveals. Chem. Asian J. 8:2330-2340

26 Arai R, Uemura S, Irie M, Matsuda K (2008) Reversible photoinduced change in molecular ordering of diarylethene derivatives at a solution-HOPG interface. J. Am. Chem. Soc. 130:9371-9379

27 Sakano T, Imaizumi Y, Hirose T, Matsuda K (2013) Formation of two-dimensional ordering of diarylethene annulated isomer upon in situ UV irradiation at the liquid/HOPG interface. Chem. Lett. 42:1537-1539

28 Yokoyama S, Hirose T, Matsuda K (2014) Phototriggered formation and disappearance of surface-confined self-assembly composed of photochromic 2-thienyl-type diarylethene: a cooperative model at the liquid/solid interface. Chem. Commun. 50:5964-5966

29 Martin RB (1996) Comparisons of indefinite self-association models. Chem. Rev. 96: 30433064

30 Yokoyama S, Hirose T, Matsuda K (2015) Effects of alkyl chain length and hydrogen bonds on the cooperative self-assembly of 2-thienyl-type diarylethenes at a liquid/highly oriented pyrolytic graphite (HOPG) interface. Chem. Eur. J. 21:13569-13576

31 Frath D, Sakano T, Imaizumi Y, Yokoyama S, Hirose T, Matsuda K (2015) Diarylethene self-assembled monolayers: cocrystallization and mixing-induced cooperativity highlighted by scanning tunneling microscopy at the liquid/solid interface. Chem. Eur. J. 21:11350-11358 